\title{
Partitioning of Molecular Weight - An Elliptic Model for Isomers
}

\section{Lemi Türker}

Department of Chemistry, Middle East Technical University, Üniversiteler, Eskişehir Yolu No: 1, 06800 Çankaya/Ankara, Turkey; e-mail: 1turker@gmail.com; lturker@metu.edu.tr

\begin{abstract}
Partitioning of any real number has been achieved based on an elliptic model introduced. Then, it has been adopted to isomeric molecules including optically active ones. Certain angles and bounds are defined. A bivariant regression model has been proposed for a set of isomeric molecules and discussed.
\end{abstract}

\section{Introduction}

Decomposition of molecules theoretically is widely used approach to facilitate some calculations in chemistry and physics [1-4]. Especially, in the past it was employed for the facile solution of Hückel molecular orbital parameters of conjugated systems prior to era of high speed computers. For that purpose group theory, graph theory etc. were the scientific approach to the solution [5-9]. The decomposition or fragmentation means to obtain smaller subgraphs from larger symmetrical molecular graphs.

On the other hand, molecular weight is a kind of mute box which does not tell much except the overall mass number and kind of atoms it possesses. It is a matter of curiosity whether it will keep its silence in the future. Although many properties of matter intensively or extensively depend on the molecular weight, these properties have not been interrelated to parts of it (in the absence of structural information including some gross and fine topology of the molecule). In the present treatment, firstly any real number has been partitioned based on an elliptic model developed. Then it has been employed partitioning of molecular weight of any set of isomeric molecules.

\section{Received: August 26, 2021; Accepted: September 24, 2021}

Keywords and phrases: isomers, molecular weight partitioning, elliptic model, optical isomers, regression.

Copyright (c) 2021 Lemi Türker. This is an open access article distributed under the Creative Commons Attribution License, which permits unrestricted use, distribution, and reproduction in any medium, provided the original work is properly cited. 


\section{Theory}

Consider a centric ellipse (see Figure 1), where $O A=a, O B=b$, are standing for major and minor hemiaxes and let $F^{\prime}$ and $F$ be the focal points. Let $M O F$ angle be $\lambda$.

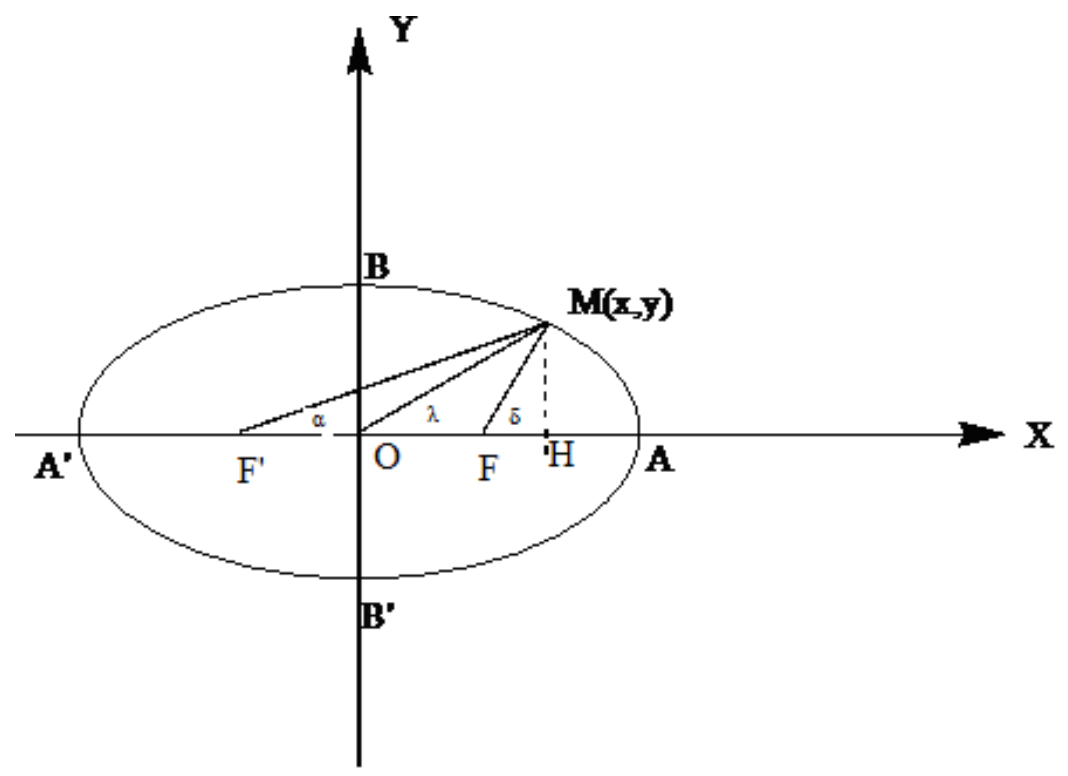

Figure 1. The ellipse of present consideration.

In $O M F$ triangle cosine theorem gives,

$$
M F^{2}=O M^{2}+O F^{2}-2 O M O F \cos \lambda .
$$

Note that in an ellipse $F^{\prime} F=2 c$ and $c^{2}=a^{2}-b^{2}[10]$. Since,

$$
M F=a-\frac{c}{a} x
$$

Inserting,

$$
x=O M \cos \lambda
$$

eq. (2) becomes,

$$
M F=a-\frac{c}{a} O M \cos \lambda .
$$

Squaring both sides of eq. (4) and equating to eq. (1) and inserting $O F=c$,

$$
a^{2}-2 c O M \cos \lambda+\frac{c^{2}}{a^{2}} O M^{2} \cos ^{2} \lambda=O M^{2}+O F^{2}-2 O M O F \cos \lambda
$$


and inserting $O F=c$, and after simplifying eq. (5) turns into,

$$
a^{2}+\frac{c^{2}}{a^{2}} O M^{2} \cos ^{2} \lambda=O M^{2}+c^{2} .
$$

Grouping the terms one gets,

$$
\begin{gathered}
\left(\frac{c^{2}}{a^{2}} \cos ^{2} \lambda-1\right) O M^{2}+a^{2}-c^{2}=0 \\
O M^{2}=\frac{\left(a^{2}-c^{2}\right) a^{2}}{a^{2}-c^{2} \cos ^{2} \lambda} .
\end{gathered}
$$

Note that $x=O M \cos \lambda$ and $y=O M \sin \lambda$. Also note that [10]

$$
\begin{aligned}
& M F^{\prime}=a+\frac{c}{a} x \\
& M F=a-\frac{c}{a} x .
\end{aligned}
$$

Then one can calculate $M F^{\prime} / M F$ ratio. Note that in an ellipse, $M F^{\prime}+M F=2 a$.

Let $c=a n$ where $n<1$. Then, the eccentricity of the ellipse becomes $c / a=n$. Substituting this value into eq. (8) and simplifying one gets,

$$
O M=\frac{\sqrt{1-n^{2}} a}{\sqrt{1-n^{2} \cos ^{2} \lambda}} .
$$

Since $x=O M \cos \lambda$, then

$$
x=\frac{\sqrt{1-n^{2}} a}{\sqrt{1-n^{2} \cos ^{2} \lambda}} \cos \lambda .
$$

Using eqs. (9) and (10),

$$
\frac{M F^{\prime}}{M F}=\frac{a+n x}{a-n x} .
$$

Adding denumerator to numerator,

$$
\frac{M F^{\prime}+M F}{M F}=\frac{2 a}{a-n x} .
$$

Substituting $M F^{\prime}+M F=M W$ and then simplifying and rearranging eq.14 becomes,

$$
M F=\frac{M W}{2 a}(a-n x) .
$$

Substituting equivalent of $x(x=O M \cos \lambda)$ one gets,

$$
M F=a\left[1-n \cos \lambda \sqrt{\frac{1-n^{2}}{1-n^{2} \cos ^{2} \lambda}}\right] .
$$


Similarly,

$$
M F^{\prime}=a\left[1+n \cos \lambda \sqrt{\frac{1-n^{2}}{1-n^{2} \cos ^{2} \lambda}}\right] .
$$

Eqs. (16) and (17) are the functions of $n$ and $\lambda$ only. Note that $F^{\prime} M H, O M H$, and $F M H$ triangles have the common height $M H$ (the ordinate $y$, see Figure 1), then the followings hold

$$
y=M F^{\prime} \sin \alpha, \quad y=M F \sin \delta, \quad y=O M \sin \lambda .
$$

Using the cosine theorem,

$$
M F^{2}-M F^{2}=2 c M F \cos \delta+2 c M F^{\prime} \cos \alpha .
$$

where angles $\delta$ and $\alpha$ are $M F H$ and $M F^{\prime} H$ angles, respectively.

\section{Estimation of $n$}

Consider Figure 2 and infinitesimally small $M O A$ triangle in which $M O A$ angle is $\lambda$. Area of the triangle is

$$
s=(O M . O A \cdot \sin \lambda) / 2 .
$$

Note that if the ellipse is sufficiently flat $(b<<a)$ the area of the triangle, $s$, is very close to area of $B O A$ sector. Apsis $X$ of point $M$ is given by $X=O M \cos \lambda$. If $\lambda$ is small enough, $O A \cong O M$, then eq. (19) becomes,

$$
s=O M^{2} \cos \lambda \sin \lambda
$$

and $O M$ can be obtained from eq. (8).

Area of BOA sector can be approximated as

$$
s \cong \frac{1}{2} \int_{0}^{\pi / 2} O M^{2} \cos \lambda \sin \lambda d \lambda
$$

Since $n<1, \cos \lambda<1$, then their multiplication has to be much smaller than 1 . Consequently, an approximate value for $O M^{2}$ is

$$
O M^{2}=\left(1-n^{2}\right) a^{2} .
$$

Inserting eq. (22) in to eq. (19), the area of the quadrant $(s=S / 4)$ be obtained as

$$
\frac{s}{4} \cong \frac{1}{2} \int_{0}^{\pi / 2}\left(1-n^{2}\right) a^{2} \cos \lambda \sin \lambda d \lambda .
$$

where $S$ is the total area of the ellipse. Evaluating the integral one obtains, 


$$
S=\left(1-n^{2}\right) a^{2} .
$$

Note that area of an ellipse is $\pi a b$, then $n$ is estimated as

$$
n \cong \sqrt{1-\frac{\pi b}{a}}
$$

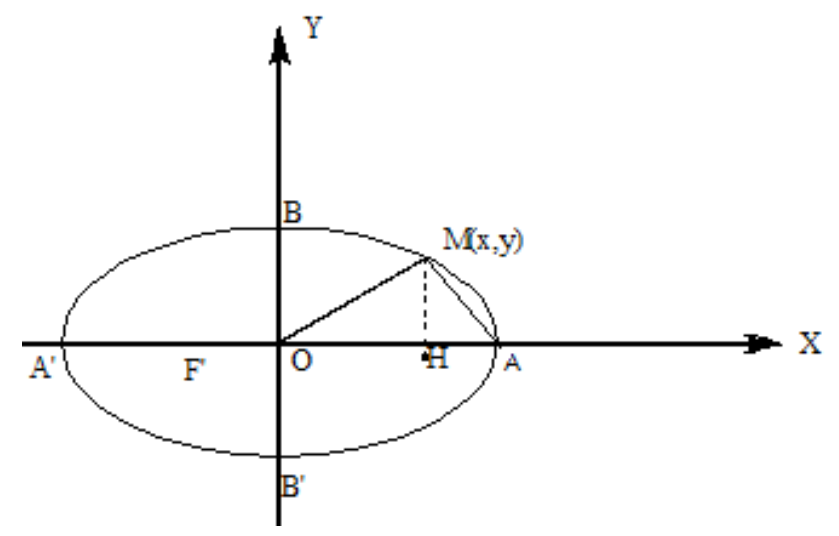

Figure 2. Helpful figure for the calculation of area of sector $A O B$.

\section{Results and Discussion}

The partitioning described above $\left(M F^{\prime} / M F\right.$ ratio) is a general approach for any number. Many partitioning alternatives mathematically exist for any number. If the model is adapted for molecules then the position of the molecule on the ellipse depends on the way of partitioning of the molecule. Since molecular weight (MW) is considered then for each set of isomeric molecules a specific ellipse has to be considered. Molecular property considered should be implicit function of angle $\lambda$, (by assumption), which is dictated by $M F$ and $M F^{\prime}$. Then the molecular property considered should be the function of those radius vectors in different contributions.

In the case of molecules by assumption $a=M W / 2$. Since $b<a$ for the existence of an ellipse $b<M W / 2$. Then $b=1$ could be taken as the lowest upper bound ( $M W$ of hydrogen is just $2 \mathrm{amu}$ ). On the other hand, $n$ to be real (see eq. (25)) it has to be $\pi b / 2 a<1$. Then, $b<2 a / \pi$ is another upper bound for $b$, namely $b<M W / \pi$.

A methodology could be established for partitioning a molecule into two parts. However, if angle $\lambda$ is associated with some physical or chemical property, then a restricted case arises. For instance, if the angle of rotation of plane polarized light is considered, then the model predicts some of the related properties (see Figure 3). 


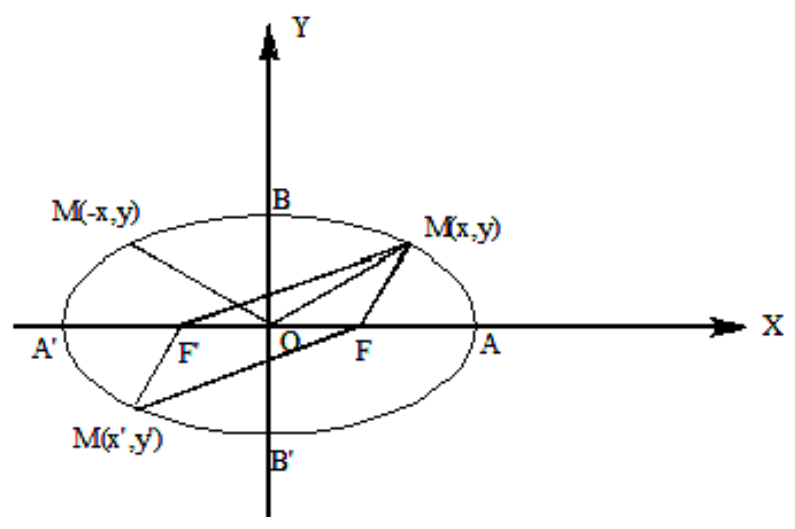

Figure 3. The relation between isomeric molecules.

Since in an ellipse $M F+M F^{\prime}=2 a$, all the isomeric molecules having molecular weight $M(M=2 a)$ reside on the ellipse, namely $M(x, y)$ and $M\left(x^{\prime}, y^{\prime}\right)$, etc., stand for isomeric compounds. Considering the first and second quadrants of the ellipse $\left(A^{\prime} B A\right.$ arc), and isomers having the molecular weight $M$ (all the achiral molecules, including meso compounds) gather at point $B(0, b)$. Whereas, chiral molecules take position in between $A$ to $(M(x, y))$ and their enantiomers along $B$ to $A^{\prime}(M(-x, y))$. On the other hand, all the meso isomers accumulate at point $B(0, b)$. For instance, inserting $\alpha=\pi-$ $\delta, \lambda$ becomes $\frac{\pi}{2}$, then eq. (8) yields $O M^{2}=a^{2}-c^{2}$ thus yields $O M=b$. Note that for meso compounds (at point $M(0, b)$ ), the model yields $M F^{\prime}=M F$, but those radius vectors are in different quadrants of the ellipse for that point. That is molecule has been partitioned into two equivalent but opposing parts. The associated polarizability values thus should cancel out each other's effect. Note that meso compound are optically inactive. Diastereomers and enantiomers occupy a position on the ellipse between points $A$ and $B$ or $B$ to $A^{\prime}$. To be more precise consider an ellipse of

$$
\frac{x^{2}}{a^{2}}+\frac{y^{2}}{b^{2}}=1
$$

Substitute $x=c$ which yields $M F$ segment (vertical focal chord), and solve for $y$ which is $y=b^{2} / a$. Angles $\alpha, \lambda, \delta$ are as shown in the Figure 4. Let $\Phi$ be,

$$
\Phi=k\left(M F^{\prime} \cos \alpha+M F \cos \delta\right),
$$

where $k$ is a constant. At point $A$ where $\alpha=\lambda=\delta=0, \Phi$ becomes 


$$
\Phi=k\left(M F^{\prime}+M F\right) .
$$

Since $M F^{\prime}+M F=2 a$, one gets

$$
\Phi=2 k a
$$

When $\lambda=\pi / 2, \alpha=\pi-\delta$ (point $B)$, then

$$
\Phi=k\left(M F^{\prime} \cos \frac{\pi}{2}+M F \cos \frac{\pi}{2}\right)
$$

which yields,

$$
\Phi=0
$$

Whereas at point $A^{\prime}$, where $\lambda=\pi, \alpha=\pi$ and $\delta=\pi, \Phi$ becomes

$$
\Phi=-2 k a .
$$

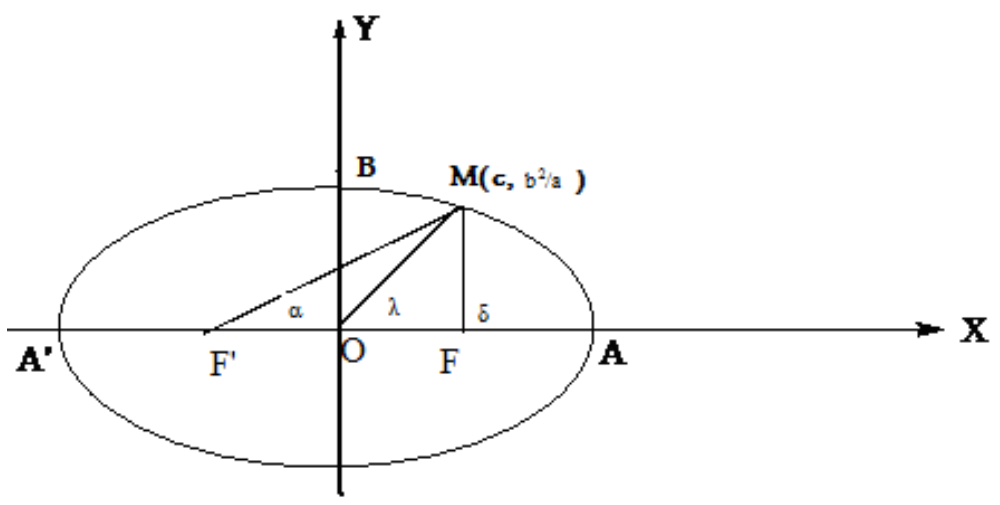

Figure 4. Location of point $M\left(c, b^{2} / a\right)$ on the ellipse.

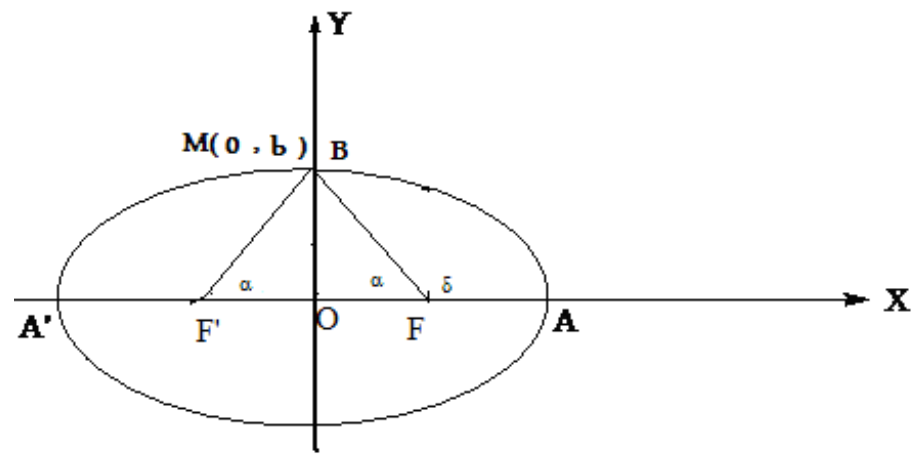

Figure 5. Location of point $M(0, b)$ on the ellipse. 
For any set of isomeric compounds, for any type of property of them various bivariant (variables $M F^{\prime}$ and $M F$ ) regression models [11] based on the elliptical model introduced presently can be developed. Note that those bivariant regression models turn into univariant type models in the case of isomers accumulated at $M\left(c, b^{2} / a\right)$.

Brewster had suggested an approach to the calculation of the sign and magnitude of optical rotation based on the consideration of two independent contributions which are mutually responsible for the observed rotation. They are (i) configuration contribution (ii) conformational contribution. For the sign of configuration contribution he proposed an empirical rule as well which is based on polarizability of the substituents around a choral center [12-15]. Although, he assumed two contributions, his way of approach is completely different from the present one.

\section{Conclusion}

The elliptic model presently introduced for the partition of molecular weight opens new horizons in physical sciences because although molecular weight appears in many scientific formulae, its introduction component wise is a brand new approach. Then many bivariant regression equations can be developed for various molecular properties to get better regression statistics compared to univariant regression equations in many cases. It would be a matter of investigation how to relate the way of partitioning of molecular weight to proper dissection of chemical structure.

\section{References}

[1] J.R. Dias, Decomposition of molecules with n-fold symmetry, in: Molecular Orbital Calculations using Chemical Graph Theory, Berlin: Springer, Heidelberg, 1993. https://doi.org/10.1007/978-3-642-77894-0_2

[2] T. Liu, M. Naderi, C. Alvin, S. Mukhopadhyay and M. Brylinski, Break down in order to build up: Decomposing small molecules for fragment-based drug design with $e$ MolFrag, J. Chem. Inf. Model. 57(4) (2017), 627-631. https://doi.org/10.1021/acs.jcim.6b00596

[3] I. Gutman, Edge-decomposition of topological indices, Iranian Journal of Mathematical Chemistry 6(2) (2015), 103-106.

[4] S.F. Vyboishchikov, A. Krapp and G. Frenking, Two complementary molecular energy decomposition schemes: The Mayer and Ziegler-Rauk methods in comparison, J. Chem. Phys. 129 (2008), 144111. https://doi.org/10.1063/1.2989805 
[5] I. Gutman and O.E. Polansky, Mathematical Concepts in Organic Chemistry, Berlin: Springer-Verlag, 1986.

[6] I. Gutman and B. Furtula, Distances in Molecular Graphs, Mathematical Chemistry Monographs-Theory 12, Distances in Molecular Graph Theory, Kragujevac: SKVER (Serbia), 2012.

[7] J.N. Murrell, S.A. Kettle and J.M. Tedder, The Chemical Bond, Chichester: Wiley, 1985.

[8] A. Streitwieser, Jr., Molecular Orbital Theory for Organic Chemists, New York: Wiley, 1961.

[9] I.S. Dimitriev, Molecules without Chemical Bonds, Moscow: Mir, 1981.

[10] M. Vygodsky, Mathematical Handbook, Moscow: Mir, 1978

[11] N. Draper and H. Smith, Applied Regression Analysis, New York: Wiley, 1966.

[12] V.M. Potapov, Stereochemistry, Moscow: Mir, 1978.

[13] J.H. Brewster, Top. Stereochemistry 2 (1967), 1-72.

[14] J.H. Brewster, Some applications of the conformational dissymmetry rule, Tetrahedron 13(2) (1961), 106-122. https://doi.org/10.1016/S0040-4020(01)92206-9

[15] E.L. Eliel, Stereochemistry of Carbon Compounds, Tokyo: McGraw-Hill Kogakusha, 1962. 